\title{
Exploration of Solar Chromosphere Using High Resolution Spectroscopic Observations
}

\author{
F. R. H. Madsen, H. S. Sawant, F. C. R. Fernandes, and J. R. Cecatto, \\ Divisão de Astrofísica - Instituto Nacional de Pesquisas Espaciais \\ Av. dos Astronautas, 1758, Prédio CEA Novo, sala 10, 12227-010, São José dos Campos, SP, Brazil
}

Received on 14 January, 2004; revised version received on 3 May, 2004

\begin{abstract}
Brazilian Solar Spectroscope (BSS), operating in the frequency range of (1000 - 2500) MHz with high time and frequency resolutions, has observed various fine structures showing intensity variations as functions of time and frequency. Here we are reporting radio bursts observed by BSS, from August to October/2001, exhibiting fine structures with total duration of the order of $300 \mathrm{~ms}$, covering a frequency range of $\sim 200 \mathrm{MHz}$. These characteristics are similar to those of the narrow band type III bursts observed above $1000 \mathrm{MHz}$. Hence these fine structures are variants of decimetric narrow band type III-like bursts. The fine structures observed exhibit variation of intensity as a function of frequency and curvatures in the frequency-time plane. These fine structures are explained as a propagation effect. We suggest that these structures are the signatures of the chromospheric density inhomogeneities lying in the line-of-sight path between the observer and the radio source. If the emitted frequencies are lower than the plasma frequency of the inhomogeneity, there will be absorption over a certain band, corresponding to the dimension of the inhomogeneity. The curved like structures are due to lower group velocities of the lower frequencies in the density inhomogeneities. The estimated scale sizes of the irregularities obtained are of the order of $10^{1}-10^{4} \mathrm{~km}$. Thus an alternative method to total solar eclipse observation is suggested for investigations of chromospheric density irregularities.
\end{abstract}

\section{Introduction}

Bhonsle et al [1] have reviewed various fine structures observed in meter and decameter wavelengths and have showed their importance in understanding of inhomogeneities in the solar corona. Various fine structures have been reported in decimeter wavelength by various authors ([2] and references therein) and many of these fine structures have been interpreted as due to various plasma mechanisms. However most of these fine structures were recorded with frequency and time resolutions of $10 \mathrm{MHz}$ and $100 \mathrm{~ms}$ respectively. For the first time we are reporting fine structures in decimeter wavelengths recorded with resolutions better than reported earlier by a factor of 2 and with a higher sensitivity of about 3 s.f.u.. The observed narrow emission gaps $\sim 3 \mathrm{MHz}$ and curvatures in the time-frequency plane at the low frequency end suggest that these effects can be due to propagation and caused by irregularities in density. Density irregularities in the chromosphere are investigated during the total solar eclipse observations and subtelescopic irregularities have been already reported in the literature [3]. These investigations of the fine structures in the decimeter wavelength can provide alternative methods to investigate density inhomogeneities in the chromosphere.

\section{Observations}

The Brazilian Solar Spectroscope - BSS - is operating over the frequency range of $1000-2500 \mathrm{MHz}$, with high time $(10-1000 \mathrm{~ms})$ and frequency $(3 \mathrm{MHz})$ resolutions, in con- junction with the polar mounted 9 meter diameter parabolic antenna. BSS is a digital spectroscope and its signal can be recorded up to 100 digital frequency channels. Absolute timing accuracy is $\sim 3 \mathrm{~ms}$ and the minimum detectable flux is $\sim 3$ s.f.u. $[4,5]$.

We have selected a sample of events showing emission gaps and curved-like structures in the time-frequency plane. The total number of selected events is 20 , of which 13 were analyzable. The data were analyzed using BSSData and BSSView softwares. The association of the fine structures with the solar activity observed with other instruments is shown in Table 1. It should be noted that most of the observed bursts exhibiting fine structures are associated with active regions near the limb. The spectra shown in the figures are examples of these events.

Figures 1a, 1b and 1c show spectra with emission gaps, corresponding to the absorption of certain frequencies, for different events. Figure 1d shows a curved-like structure at the lower frequencies. Figures $1 \mathrm{~b}$ and $1 \mathrm{c}$ show also delays in the arrival of the lower and higher frequencies respectively, but these delays are in accordance with the normal drift rates observed in type III bursts above $100 \mathrm{MHz}$ [6] and are not due to propagation effects.

\section{Interpretations}

Meléndez et al [6] have investigated in detail characteristics of the decimetric type III bursts above $1000 \mathrm{MHz}$. Total duration and bandwidth of the bursts in investigations are similar to decimetric narrow band type III bursts observed 
TABLE 1 . Type of spectral activity associated with the analyzed events.

\begin{tabular}{ccccccc}
\hline Date & Time (UT) & & Active & Spectral Type / & Observatory \\
& Start & Max. & End & Region & Class & \\
\hline & $15: 02$ & $15: 04$ & $15: 23$ & S16E80 & H-alpha & Ramey Air Base \\
& $15: 02$ & $15: 04$ & $15: 23$ & S17E77 & H-alpha & Holloman AFB \\
08/22/2001 & $15: 04$ & $15: 04$ & $15: 34$ & S16E84 & H-alpha & Ramey Air Base \\
& $15: 02$ & $15: 06$ & $15: 10$ & S17E77 & X-Ray / C3.7 & GOES \\
& $15: 13$ & - & $15: 16$ & - & Radio / DCIM & Ondrejov \\
\hline \multirow{3}{*}{$09 / 13 / 2001$} & $13: 05$ & $13: 05$ & $13: 10$ & S15W10 & H-alpha & Ramey Air Base \\
& $13: 03$ & $13: 06$ & $13: 07$ & S15W10 & X-Ray / C2.4 & GOES \\
& $13: 03$ & - & $13: 06$ & - & Radio / DCIM & Ondrejov \\
\hline & $15: 33$ & $15: 36$ & $16: 02$ & N15E49 & H-alpha & Holloman AFB \\
& $15: 34$ & $15: 39$ & $16: 20$ & N14E50 & H-alpha & Ramey Air Base \\
$09 / 18 / 2001$ & $17: 06$ & $17: 06$ & $17: 13$ & S18E85 & H-alpha & Holloman AFB \\
& $15: 49$ & $15: 52$ & $15: 54$ & - & X-Ray / M1.0 & GOES \\
& $15: 50$ & - & $15: 54$ & - & Radio / DCIM & Ondrejov \\
& $15: 50$ & - & $15: 54$ & - & Radio / Type III & Blen \\
\hline
\end{tabular}
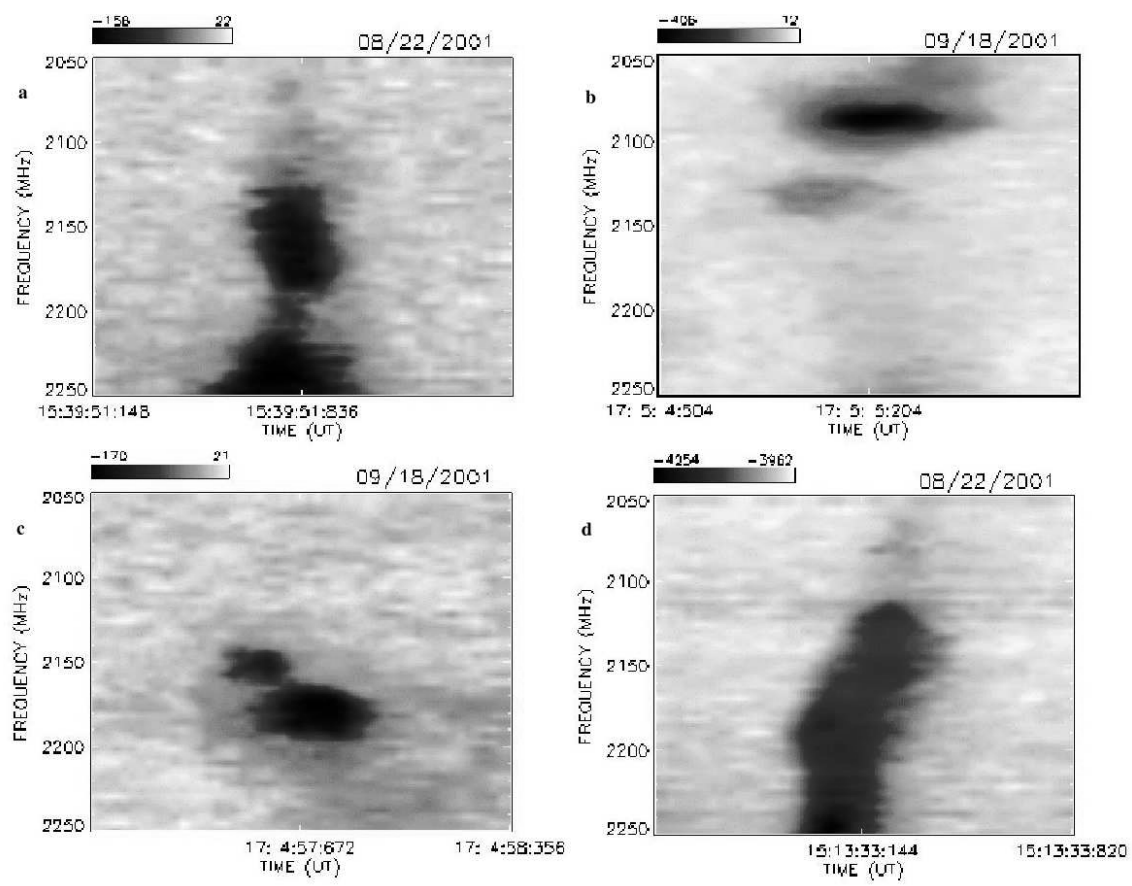

Figure 1. Radio bursts with emission gaps $\sim 20 \mathrm{MHz}$, as seen in black color superimposed on the orange background almost at the center of each frame, for (a) August 22, 2001, (b) September 9, 2001, and (c) September 18, 2001. (d) Radio burst exhibiting curvature at the low frequencies with the same color code as above, for August 22, 2001.

above $1000 \mathrm{MHz}$. This leads us to suggest that the emission gaps and curvatures at the low frequency ends observed in these type III-like bursts are due to propagation effects caused by the inhomogeneities in the line of sight between the radio source and the observer. Thus emission gaps can be associated with the scale length of the density irregularities transverse to the line-of-sight.

When there is an inhomogeneity in density lying in the line-of-sight path between the observer and the radio source (as shown schematically in Fig. 2), there will be absorption over a certain band of frequencies (Fig. 1a, b and c). Knowing the absorption band, and assuming emission at the second harmonic due to beam plasma interaction one can estimate the scale size of the irregularity, assuming that the inhomogeneities are close enough to the sources and using an electron density vs. height model [7].

On the other hand, the curved-like structures (Fig. 1d) are due to the dispersion of the propagating waves through an inhomogeneity (also shown in Fig. 2), as the lower frequencies propagate with a lower group velocity. Using the relationship between the group path delay and the columnar electron density, one can obtain the length of the irregularities along the line-of-sight. 


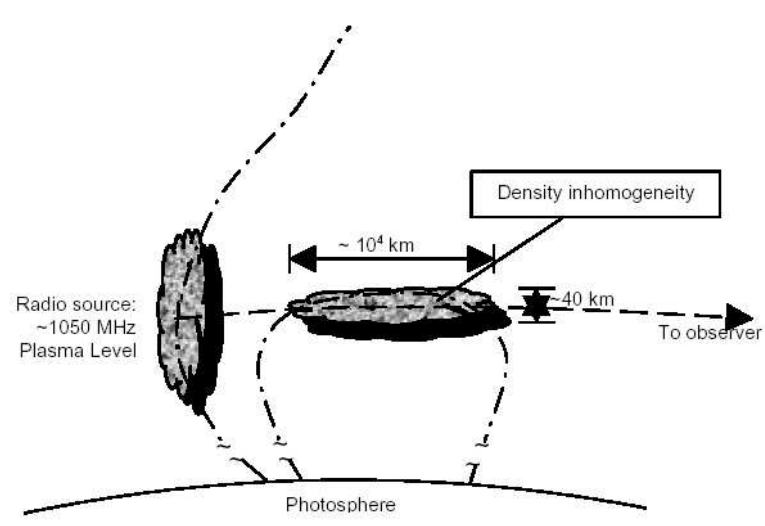

Figure 2. Schematic view of the possible geometry (not to scale) of the chromospheric electron density irregularities. The excess in density is assumed to be $3 \%$. The magnetic field lines are shown by dash-dotted lines and the exciter beam is assumed to travel along the open field line.

\section{Calculations}

\subsection{Scale sizes of the inhomogeneities associ- ated with emission gaps}

Assuming emission at the second harmonic of the plasma frequency the densities are obtained corresponding to absorption band in the frequency by using the following equation:

$$
f_{p}=9 \times 10^{-3} \sqrt{N_{e}} M H z
$$

where $N_{e}$ is the electron number density in $\mathrm{cm}^{-3}$. Using the estimated densities, the height of the source above the photosphere is obtained using the Cilié \& Menzel model [7] for the electronic (chromospheric) densities in relation to the height:

$$
N_{e}(h)=10 \times 5.7 \times 10^{11} e^{-7.7 \times 10^{-4}(h-500)} \mathrm{cm}^{-3}
$$

where $h$ is in kilometers, and the 10 factor is due to the maximum of the solar cycle. Densities in the chromosphere and corona vary by a factor of $\sim 10$ from minimum to maximum during the solar cycle. Since these observations are during the solar cycle maximum, it is appropriate to include a factor of 10 in the above equation. The difference in the height corresponding to the absorption band as estimated above is the transverse dimension of the irregularity in the line of sight between the source and the observer.

The results obtained for the scale sizes of these irregularities are shown in Table 2, where the frequencies $f_{s}$ and $f_{e}$ are the starting and the end frequencies of the emission gap; the heights $h_{f_{s}}$ and $h_{f_{e}}$ are obtained given $f_{s}$ and $f_{e}$; and $L$, the difference $h_{f_{e}}-h_{f_{s}}$, is the obtained length of the inhomogeneities, transverse to the line-of-sight.

\subsection{Dimension of the inhomogeneities associ- ated with propagation delays}

If density inhomogeneities lie in the line-of-sight path between the source and the observer with an excess in density $\sim 3 \%$ of the ambient densities, the lower frequencies will propagate with lower group velocities and hence delays will be observed in comparison to the higher frequencies resulting in the observed curved-like structures in the frequency-time plane. Thus by using the relationship between time delay and densities [8], the length of irregularities in the line of sight can be estimated:

$$
\Delta t=\frac{b}{c \omega^{2}} \int_{0}^{l} N d l
$$

where $N$ is the number density of free electrons, $b=$ $1.6 \times 10^{3} \mathrm{MKS}$ units, $\omega$ is the angular frequency, $c$ is the speed of light in vacuum and $l$ is the length of the inhomogeneity.

The obtained results for the length of these inhomogeneities are shown in Table 3, where $f_{a v g}$ is the average frequency in the curved-like structures; $N_{f_{a v g}}$ is the local ambient density of the medium; $\Delta N$ is the density of the inhomogeneity, which is about $3 \%$ more than $N_{f_{a v g}} ; \Delta t$ is the propagation delay of the lower frequencies; and $l$ is the obtained dimension of the inhomogeneity along the line-ofsight.

\section{Conclusions}

Similar results have been obtained in the meter-decameter wavelengths by various researchers as reviewed by Bhonsle et al. [1]

1) Various types of fine structures in the decimetric range suggest the presence of sub telescopic scale density inhomogeneities in the chromosphere.

2) The dimensions of these inhomogeneities transverse to the line-of-sight are in the range of $10^{1}-10^{2}$ $\mathrm{km}$ and they are associated with the fine structures showing variation of intensity as a function of frequency.

3) The dimensions of these irregularities along the lineof-sight are $\sim 7 \times 10^{4} \mathrm{~km}$. They are associated with propagation delays, assuming that the inhomogeneities have density excesses $\sim 3 \%$ of the ambient density.

4) Thus investigations of solar dynamic spectra of the solar bursts in decimeter wavelength with high sensitivity and high resolutions are an alternative to solar eclipse observations for investigations of solar chromosphere. 
TABLE 2. Length of the inhomogeneities associated with emission gaps

\begin{tabular}{ccccccc}
\hline Date & Start time $(\mathrm{UT})$ & $f_{s}(\mathrm{MHz})$ & $h_{f_{s}}(\mathrm{~km})$ & $f_{e}(\mathrm{MHz})$ & $h_{f_{e}}(\mathrm{~km})$ & $L(\mathrm{~km})$ \\
\hline $08 / 22 / 2001$ & $15: 39: 49$ & 2210 & $8.209 \mathrm{E}+03$ & 2182 & $8.242 \mathrm{E}+03$ & 33 \\
$09 / 13 / 2001$ & $13: 05: 35$ & 2126 & $8.309 \mathrm{E}+03$ & 2090 & $8.353 \mathrm{E}+03$ & 44 \\
$09 / 18 / 2001$ & $15: 50: 19$ & 2094 & $8.348 \mathrm{E}+03$ & 2066 & $8.383 \mathrm{E}+03$ & 35 \\
$09 / 18 / 2001$ & $17: 04: 57$ & 2166 & $8.260 \mathrm{E}+03$ & 2154 & $8.275 \mathrm{E}+03$ & 15 \\
$09 / 18 / 2001$ & $17: 05: 05$ & 2118 & $8.318 \mathrm{E}+03$ & 2106 & $8.333 \mathrm{E}+03$ & 15 \\
$09 / 18 / 2001$ & $17: 05: 12$ & 2170 & $8.256 \mathrm{E}+03$ & 2138 & $8.294 \mathrm{E}+03$ & 38 \\
$09 / 18 / 2001$ & $17: 05: 27$ & 2166 & $8.260 \mathrm{E}+03$ & 2134 & $8.299 \mathrm{E}+03$ & 39 \\
$09 / 18 / 2001$ & $17: 05: 33$ & 2202 & $8.217 \mathrm{E}+03$ & 2162 & $8.265 \mathrm{E}+03$ & 48 \\
$10 / 01 / 2001$ & $14: 59: 01$ & 2094 & $8.348 \mathrm{E}+03$ & 2086 & $8.358 \mathrm{E}+03$ & 10 \\
$10 / 01 / 2001$ & $15: 00: 36$ & 2202 & $8.217 \mathrm{E}+03$ & 2126 & $8.309 \mathrm{E}+03$ & 92 \\
\hline
\end{tabular}

TABLE 3. Length of the inhomogeneities associated with propagation delays.

\begin{tabular}{ccccccc}
\hline & & \multicolumn{2}{c}{ Density } \\
Date & Start time $(\mathrm{UT})$ & $f_{\text {avg }}(\mathrm{MHz})$ & $N_{f_{\text {avg }}}\left(\mathrm{cm}^{-3}\right)$ & $\Delta N\left(\mathrm{~cm}^{-3}\right)$ & $\Delta t(\mathrm{~s})$ & $l(\mathrm{~km})$ \\
\hline $08 / 22 / 2001$ & $15: 13: 33$ & 2158 & $1.437 \mathrm{E}+10$ & $1.480 \mathrm{E}+10$ & 0.223 & $1.298 \mathrm{E}+05$ \\
$09 / 13 / 2001$ & $13: 05: 24$ & 2123 & $1.391 \mathrm{E}+10$ & $1.433 \mathrm{E}+10$ & 0.088 & $5.123 \mathrm{E}+04$ \\
$09 / 13 / 2001$ & $13: 05: 26$ & 2166 & $1.448 \mathrm{E}+10$ & $1.491 \mathrm{E}+10$ & 0.086 & $5.006 \mathrm{E}+04$ \\
\hline
\end{tabular}

\section{Acknowledgement}

We would like to thank Dr. M. Aschwanden for useful discussions and suggestions for the interpretations of the decimetric fine structures.

\section{References}

[1] H. S. Bhonsle, H. S. Sawant, and S. S. Degaonkar, Space Science Reviews, 24, 259 (1979).

[2] F. C. R. Fernandes, Catálogo de Espectros Dinâmicos de Explosões Solares Decimétricas Registradas pelo Brazilian Solar Spectroscope (BSS) (2001).

[3] L. N. Kurochka, O. T. Matsuura, and E. Picazzio, Solar Physics, 170, 227 (1997).
[4] H. S. Sawant, K. R. Subramanian, C. Faria, F. C. R. Fernandes, J. H. A. Sobral, J. R. Cecatto. R. R. Rosa, H. O. Vats, J. A. C. F. Neri, E. M. B. Alonso, F. P. V. Mesquita, V. A. Portezani, and A. R. F. Martinon, Solar Physics, 200, 167 (2001).

[5] H. S. Sawant, K. R. Subramanian, C. Faria, S. Stephany, F. C. R. Fernandes, J. R. Cecatto, R. R. Rosa, V. A. Portezani, F. P. V. Mesquita, and E. M. B. Alonso, Astronomical Soceity of the Pacific Conference Series, 206, 347 (2000).

[6] J. L. Meléndez, H. S. Sawant, F. C. R. Fernandes, and A. O. Benz, Solar Physics, 187, 77 (1999).

[7] G. G. Cilié and D. H. Menzel, Harvard College Observatory Circular, 410 (1935).

[8] R. S. Lawrence, C. G. Little, and H. J. A. Chivers, Proc. IEEE, U.S.A. 52, 4 (1964). 